\title{
Relationships Among Resistances to Fusarium and Aspergillus Ear Rots and Contamination by Fumonisin and Aflatoxin in Maize
}

\author{
Leilani A. Robertson-Hoyt, Javier Betrán, Gary A. Payne, Don G. White, Thomas Isakeit, \\ Chris M. Maragos, Terence L. Molnár, and James B. Holland
}

First author: Department of Plant Pathology and Department of Crop Science, North Carolina State University, Raleigh 27695-7620; second author: Department of Soil and Crop Sciences, Texas A\&M University, College Station 77843-2474; third author: Department of Plant Pathology, North Carolina State University, Raleigh 27695-7567; fourth author: Department of Crop Sciences, University of Illinois, Urbana-Champaign 61801; fifth author: U.S. Department of Agriculture-Agricultural Research Service (USDA-ARS) National Center for Agricultural Utilization Research, Peoria, IL, 61604; sixth author: Department of Plant Pathology and Microbiology, Texas A\&M University, College Station; seventh author: Pioneer Génétique, Pacé, France; and eighth author: USDA-ARS, Plant Science Research Unit, Department of Crop Science, North Carolina State University, Raleigh 27695-7620.

Accepted for publication 30 September 2006.

\section{ABSTRACT}

Robertson-Hoyt, L. A., Betrán, J., Payne, G. A., White, D. G., Isakeit, T., Maragos, C. M., Molnár, T. L., and Holland, J. B. 2007. Relationships among resistances to Fusarium and Aspergillus ear rots and contamination by fumonisin and aflatoxin in maize. Phytopathology 97:311-317.

Fusarium verticillioides, F. proliferatum, and Aspergillus flavus cause ear rots of maize and contaminate the grain with mycotoxins (fumonisin or aflatoxin). The objective of this study was to investigate the relationships between resistance to Fusarium and Aspergillus ear rots and fumonisin and aflatoxin contamination. Based on a previous study of 143 recombinant inbred lines from the cross NC300 $\times$ B104, 24 lines with the highest and 24 lines with the lowest mean fumonisin concentration were selected for further evaluation. Paired plots of each line were inoculated with $F$. verticillioides and $F$. proliferatum or with A. flavus in replicated trials in 2004 and 2005 in Clayton, NC, and College Station, TX. The low-fumonisin group had significantly lower levels of fumonisin, aflatoxin, and Fusarium and Aspergillus ear rots. Across year-location environments, all four traits were significantly correlated; the genotypic correlation $\left(\mathrm{r}_{\mathrm{G}}\right)$ ranged from $\mathrm{r}_{\mathrm{G}}=0.88$ (aflatoxin and Aspergillus ear rot) to $\mathrm{r}_{\mathrm{G}}=0.99$ (Fusarium and Aspergillus ear rots). Quantitative trait loci (QTLs) were identified and their effects estimated. Two QTLs affected both toxin concentrations, one QTL affected both ear rots, and one QTL affected Aspergillus and Fusarium rots and fumonisin. These results suggest that at least some of the genes involved in resistance to ear rots and mycotoxin contamination are identical or genetically linked.

Additional keyword: corn.
Mycotoxins in maize, such as fumonisins, produced by Fusarium spp., and aflatoxins, produced by Aspergillus spp., are a concern due to livestock poisoning $(10,26)$ and cancer and birth defects in humans $(4,11,12,22)$. When conditions for fungal growth are optimum, there are no cultural practices that are sufficient to keep mycotoxin levels below Food and Drug Administration limits and suggestions for safe toxin levels (5-7). Genetic resistance is the best defense against mycotoxin contamination. However, at present, no commercial hybrids are completely resistant to mycotoxin contamination.

Both fumonisin and aflatoxin can be found on the same maize ear, but there is debate over whether Fusarium and Aspergillus spp. are antagonistic pathogens. Marín et al. (16) found that the growth of Aspergillus spp. was slowed in the presence of Fusarium spp., suggesting a competitive relationship and a negative correlation between the growth of these two species, perhaps due to competition for the same resources or niche environment on maize ears. Similarly, Zummo and Scott (35) found lower levels of aflatoxin in grain of plants inoculated with both Fusarium spp. and Aspergillus flavus (Link:Fr.) than in grain of plants inoculated with A. flavus alone. They concluded that Fusarium spp. are able to inhibit A. flavus growth, leading to

Corresponding author: J. B. Holland; E-mail address: james_holland@ncsu.edu

DOI: 10.1094/PHYTO-97-3-0311

This article is in the public domain and not copyrightable. It may be freely reprinted with customary crediting of the source. The American Phytopathological Society, 2007. lower aflatoxin levels. These pathogens may require similar substrates for growth and development, and also may have similar interactions with the host plant. If this were true, host plant resistance to one pathogen species would be associated with resistance to the other. It is possible that the genes responsible for common resistances could be the same or genetically linked. Wisser et al. (34) reported evidence that quantitative trait loci (QTLs) conferring resistance to different diseases are clustered in the maize genome.

In contrast, however, Ono et al. (18) found that fumonisin and aflatoxin were both found in Brazilian corn, although fumonisin was the more prevalent mycotoxin. Moreover, Abbas et al. (1) found that, across test environments in hybrids naturally infected with both Fusarium spp. and A. flavus, aflatoxin and fumonisin levels were positively correlated. These results suggest that both species can thrive on the same plant without necessarily competing for the same resources if host plants are highly susceptible. The positive correlation between the two mycotoxins suggests that some mechanisms of resistance to the fungi may be shared, but this cannot be determined with certainty without partitioning the genetic components from the total phenotypic correlation (23). To our knowledge, neither genetic correlations between Fusarium ear rot, Aspergillus ear rot, and fumonisin and aflatoxin concentration, nor shared mechanisms of resistance among ear rots, have been demonstrated.

Due to the limited understanding of the relationship between A. flavus and Fusarium spp. infections in corn and the lack of understanding of common or distinct resistance mechanisms to 
different ear rot pathogens, the objective of this study was to estimate the correlations between Fusarium and Aspergillus ear rot, fumonisin, and aflatoxin contamination in maize. To minimize the confounding effects of interactions between pathogen species on the evaluation of host plant resistance, we inoculated plants with only one pathogen or the other.

\section{MATERIALS AND METHODS}

Population development. NC300 was identified in preliminary studies as a potential source of resistance to Fusarium ear and kernel rot and low fumonisin contamination (23). A segregating population, derived from the cross $\mathrm{NC} 300 \times \mathrm{B} 104$, was created. Inbred NC300 (derived from the tropical hybrids Pioneer X105A, Pioneer X306B, and H5) was crossed to inbred B104 (derived from the BS13 strain of Iowa Stiff Stalk Synthetic). A random sample of $F_{2}$ plants from this cross were self-pollinated to form $\mathrm{F}_{2: 3}$ families. $\mathrm{F}_{2: 3}$ families were grown ear-to-row, and a single self-fertilized ear was harvested from each row without selection. This procedure was repeated until $143 \mathrm{~F}_{6}$-derived recombinant inbred lines (RILs) were developed. $\mathrm{F}_{7}$ or $\mathrm{F}_{8}$ generation seed were used in this study to represent the RILs. Robertson et al. (23) evaluated this population in replicated trials that were artificially inoculated with Fusarium verticillioides (Sacc.) Nirenberg (synonym $F$. moniliforme J. Sheld.), and $F$. proliferatum (T. Matsushima) Nirenberg, in three environments. Mean fumonisin concentration was estimated for each line, and the 24 lines with highest mean fumonisin concentration and 24 lines with lowest mean fumonisin concentration were selected for further study. These two groups of lines will be referred to as the high and low fumonisin groups, respectively, based on their performance in the previous trials reported by Robertson et al. (23).

Field evaluation. The 24 high-fumonisin lines, 24 lowfumonisin lines, with the two parental lines, NC300 and B104, and six check inbreds (CML176, CML269, CML288, FR2128, TX110, and TX770) comprised a total of 56 experimental entries for the study. The experiment was conducted in 2004 and 2005 in two locations: Clayton, NC, and College Station, TX. The experimental design was a split-plot design where whole plots were genotypes and subplots were pathogen inoculations (Fusarium or Aspergillus spp.). The whole plots were arranged following 7-by-8 $\alpha$-lattice designs replicated three times in each environment. Whole plots were two-row plots, and each subplot was one row, which was inoculated with either Fusarium spp. or with A. flavus. Plots were $3.048 \mathrm{~m}$ in length with $0.9144 \mathrm{~m}$ between plots in both locations. Plots were overplanted and thinned to $\approx 20$ plants. Each subplot row was tagged before inoculation to indicate which inoculation treatment was to be applied.

All plants in the Fusarium subplot rows were inoculated with three isolates each of $F$. verticillioides and $F$. proliferatum: $F$. verticillioides isolates ISU95082, ISU94445, and ISU94040 and F. proliferatum isolates $310,37-2$, and 19 . This mixture of isolates was created to represent the heterogeneity of pathogen species in the natural environment (9). The Fusarium isolates were removed from glycerol storage at $-80^{\circ} \mathrm{C}$ and cultured separately on potato dextrose agar. Inoculum was prepared by washing conidia from the cultures with $0.05 \%$ Triton X-100 (Fisher Biotech, Fairlawn, $\mathrm{NJ}$ ) and diluting the resulting conidial suspension of the six isolates to a final concentration of $\approx 1 \times 10^{6}$ conidia ml ${ }^{-1}$.

In order to reduce the chance of escapes, two inoculations of Fusarium spp., 1 week apart, were performed. At each inoculation, the primary ear of 10 plants in one row (one subplot) of each two-row whole plot was inoculated with $10 \mathrm{ml}$ of inoculum. Silk channel inoculation (injecting inoculum into the silk channel while the silks are fresh) was performed $\approx 10$ days after mean silking date within each location, and direct ear inoculation (injecting inoculum directly through the husk onto the ear) was performed 7 days later (9).
A. flavus inoculum was prepared using isolate NRRL 3357 $(32,33)$. The isolate was cultured on autoclaved corn kernels, and inoculum was prepared by washing conidia from the kernels with 2 to 3 drops of Tween-20 (Fisher Biotech) per $100 \mathrm{ml}$ of water and diluting the resulting conidial suspension to a final concentration of $\approx 1 \times 10^{7}$ conidia $\mathrm{ml}^{-1}$. One inoculation was performed $\approx 10$ days after mean silking date within each location, using silk channel inoculation. The primary ear of 10 plants in one subplot of each whole plot was inoculated with $3 \mathrm{ml}$ of inoculum.

When all plants had reached physiological maturity, primary ears were hand harvested and air dried to $\approx 14 \%$ moisture. After drying, ears on the Fusarium spp.-inoculated row were rated by visually identifying the percentage of kernels exhibiting Fusarium ear rot symptoms on each inoculated ear. Ears on the A. flavusinoculated row were rated by visually identifying the percentage of kernels exhibiting Aspergillus ear rot symptoms on each inoculated ear. The grain from all inoculated ears within each individual row of a plot then was shelled, bulked, and ground to a fine particle size using a Romer mill (series II mill; Romer Labs Inc., Union, MO). Fumonisin concentrations were measured on $25 \mathrm{~g}$ of ground grain from each Fusarium spp. subplot. Fumonisin concentrations were measured in triplicate for each sample using enzyme-linked immunosorbent assay (9). Aflatoxin concentrations were obtained from a 50-g subsample of ground grain from each Aspergillus spp. subplot using the VICAM Aflatest (VICAM, Watertown, MA).

Statistical analysis. Analysis of variance was conducted for each trait (fumonisin concentration, aflatoxin concentration, Fusarium ear rot, and Aspergillus ear rot) and least square means for each line were estimated with the PROC MIXED procedure from SAS (27). Because each trait was measured on only one subplot of each whole plot, the analysis was conducted on the basis of the lattice design of whole plots. Line was considered a fixed effect. Environment (year-location), replication within environment, incomplete block within replication-environment, and environment-line interaction were considered random effects. Tests for significance of genotype-environment interaction were performed with a $\chi^{2}$ test of the difference between -2 times the logs of the restricted maximum likelihoods of the complete model and a reduced model lacking the genotype-environment interaction (15). The approximate $P$ value of the test was obtained by dividing the $P$ value of the one degree of freedom $\chi^{2}$ statistic by two $(15,28) . F$ tests using the CONTRAST statement of PROC MIXED from SAS (27) were used to compare the means of line groups chosen based on high and low levels of fumonisin for all four traits measured. Genotypic and phenotypic correlations among Fusarium ear rot, Aspergillus ear rot, fumonisin, and aflatoxin were estimated using multivariate REML in PROC MIXED of SAS (13). Standard errors of genotypic and phenotypic correlations obtained with multivariate analyses were obtained with the delta method (14).

Genotyping and linkage map construction. Four plants per RIL and parental line were sampled for extraction. The whole population, with the exception of 1 of the 143 lines, was genotyped. DNA extractions were performed using DNeasy Plant Mini Kit system (Qiagen, Inc., Valencia, CA). Simple sequence repeat (SSR) primers were selected for screening using consensus map information from the Maize Genetics and Genomics Database (available online) to reference location of marker loci. Parental lines were screened with SSR primers to identify polymorphisms (29). Polymerase chain reaction (PCR) products were separated by electrophoresis in $4 \%$ super fine resolution (SFR) agarose gels (Amresco, Solon, $\mathrm{OH}$ ) and visualized by staining gels with $0.05 \%$ ethidium bromide and exposing them to ultraviolet light.

Locus orders and recombination frequencies were estimated using multipoint mapping in MapmakerEXP (Whitehead Institute, Cambridge, MA). Recombination frequencies then were transformed to centimorgans (cM) using Haldane's mapping function 
in MapmakerEXP. Segregation distortion was tested with $\chi^{2}$ tests using Windows QTL Cartographer version 2.5 (31).

QTL detection and estimation. QTLs were detected using only the phenotype data obtained in this study (the 24 highest and 24 lowest lines from the original population). Composite interval mapping (CIM) was implemented in Windows QTL Cartographer version 2.5 to provide initial models for the multiple interval mapping (MIM) procedure. For CIM, the forward regression method was used for cofactor selection and a $10-\mathrm{cM}$ window size was used for the genome scans. The threshold log of the likelihood ratio score to declare significance at $P=0.05$ was estimated empirically with 300 permutations (8). QTL analysis was performed across all year-location environments.

QTL peak positions from the CIM analysis (with a log of the likelihood ratio threshold value of 2.0 and minimum $5-\mathrm{cM}$ minimum between QTLs) were used as the initial models for MIM in Windows QTL Cartographer 2.5. MIM models were created and tested in an iterative, stepwise fashion, searching for new QTLs to add to the current model, and testing their significance after each search cycle. New models were accepted if they decreased the Bayesian Information Criterion (BIC) (21). The BIC favors models with higher likelihoods, but includes a penalty for each additional parameter added to the model to help prevent overfitting the models. After no additional QTLs could be added to a model according to the BIC, each pair of QTLs in the model was tested for epistatic interactions. Epistatic interactions were maintained in the model if they decreased the BIC. The model with minimum BIC was chosen and QTL effects were estimated simultaneously using the "summary" option. The proportion of phenotypic variation explained by each QTL was estimated from the simultaneous fitting of QTL positions in the final model by Windows QTL Cartographer. When comparing QTLs for two or more traits, QTL positions were considered to be overlapping when they were within $20 \mathrm{cM}(3,17,30)$.

\section{RESULTS}

Field evaluations. We rated the Aspergillus spp.-inoculated plots for Aspergillus ear rot and very rarely observed Fusarium ear rot on those ears, suggesting that substantial levels of $\mathrm{Fu}$ sarium spp. growth were not observed in the Aspergillus spp.inoculated plots. Fusarium spp.-inoculated plots also rarely exhibited Aspergillus ear rot. Thus, there is no indication that fungal competition was an important factor in this study.

Lines differed significantly $(P<0.0001)$ for aflatoxin and fumonisin concentrations, as well as for Fusarium and Aspergillus ear rots, across all year-location environments. The means of the parent lines, NC300 and B104, across all year-location environments, differed significantly only for Aspergillus ear rot (Table 1) in agreement with Robertson et al. (23), who reported that these parent lines exhibited similar levels of resistance to Fusarium ear rot and fumonisin contamination, although their progeny exhibited significant transgressive segregation for susceptibility to both aspects of this disease. None of the checks differed significantly for aflatoxin or fumonisin concentration, although FR2128 did have four times the amount of aflatoxin as Tx110 and more than three times the amount of fumonisin as all of the other check lines (Table 1). None of the checks were significantly different for either Aspergillus or Fusarium ear rot.

Means for aflatoxin levels across year-location environments ranged among RILs from 261 to $4,876 \mathrm{ng} \mathrm{g}^{-1}$, and fumonisin levels ranged from 4 to $111 \mu \mathrm{g} \mathrm{g}^{-1}$ (Table 2). Aspergillus ear rot levels across year-location environments ranged among RILs from 9 to $73 \%$ and, for Fusarium ear rot, averages ranged from 8 to $85 \%$ (Table 2). For each trait, the five lowest-ranking lines across year-location environments were always from the selected low-fumonisin group, and the highest-ranking five lines were from the selected high-fumonisin group, with one exception (Table 2). The one exception was RIL-48, which was in the selected low-fumonisin group and ranked among the top five lines for high mean aflatoxin concentration, and ranked near the middle for fumonisin concentration and both ear rots (Table 2).

Genotype-environment interaction was significant for all traits $(P<0.0001)$. Therefore, we also compared trait means for parents, checks, and high- and low-fumonisin groups within each of the four year-location environments. The group of 24 RILs selected for high fumonisin levels had significantly higher levels of fumonisin, aflatoxin, and Fusarium and Aspergillus ear rots in all environments (Table 3). The magnitude of the difference between low- and high-fumonisin groups differed among yearlocation environments, contributing to the genotype-environment interaction variance. Ranks of individual checks also varied across year-location environments (Table 3). Across year-location environments, the group of 24 RILs selected for high fumonisin levels had significantly $(P<0.0001)$ higher levels of fumonisin, aflatoxin, and Fusarium and Aspergillus ear rots than the group of RILs selected for low fumonisin concentration (Table 1).

Genotypic and phenotypic correlations. Genotypic and phenotypic correlations among all four traits were significant and high (Table 4). Genotypic correlation estimates ranged from $\mathrm{r}_{\mathrm{G}}=$ 0.88 (standard error $[\mathrm{SE}]=0.06$ ) between aflatoxin and Aspergillus ear rot to $\mathrm{r}_{\mathrm{G}}=0.99(\mathrm{SE}=0.01)$ between Fusarium ear rot and Aspergillus ear rot. The phenotypic correlation estimates ranged from $r_{P}=0.53(\mathrm{SE}=0.06)$ between aflatoxin and fumonisin concentration to $\mathrm{r}_{\mathrm{P}}=0.81(\mathrm{SE}=0.03)$ between Fusarium ear rot and Aspergillus ear rot. Genotypic correlations were higher than their corresponding phenotypic correlation for all

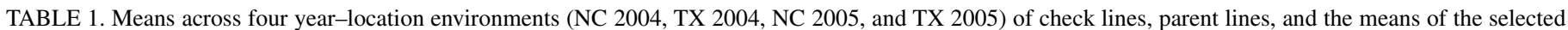
high- and low-fumonisin groups for aflatoxin, Aspergillus ear rot, fumonisin, and Fusarium ear $\operatorname{rot}^{\mathrm{a}}$

\begin{tabular}{|c|c|c|c|c|}
\hline Lines & Aflatoxin (ng g $\left.{ }^{-1}\right)$ & Aspergillus ear rot (\%) & Fumonisin $\left(\mu \mathrm{g} \mathrm{g}^{-1}\right)$ & Fusarium ear rot $(\%)$ \\
\hline \multicolumn{5}{|l|}{ Check and parent lines } \\
\hline CML176 & 298 & 6 & 10 & 10 \\
\hline CML269 & 586 & 10 & 9 & 12 \\
\hline CML288 & 576 & 21 & 5 & 12 \\
\hline FR2128 & 1,034 & 21 & 31 & 26 \\
\hline TX110 & 247 & 21 & 10 & 25 \\
\hline TX770 & 742 & 12 & 8 & 22 \\
\hline NC300 & 122 & 4 & 3 & 7 \\
\hline B104 & 643 & 25 & 10 & 24 \\
\hline Mean of high-fumonisin lines & 2,422 & 45 & 62 & 50 \\
\hline Mean of low-fumonisin lines & 849 & 17 & 11 & 18 \\
\hline High-low-fumonisin lines & $1,573 * * * *$ & $28 * * * *$ & $51 * * * *$ & $32 * * * *$ \\
\hline $\operatorname{LSD}(0.05)^{\mathrm{b}}$ & 1,629 & 21 & 30 & 20 \\
\hline
\end{tabular}

a Asterisks (****) indicate significance at $P<0.0001$.

b Least significant differences (LSDs) are appropriate for comparing any two line means. 
pairs of traits. The high correlations were evident in the consistency of RIL ranks across traits (Table 2). Three RILs ranked among the lowest five RILs for at least three traits, and two RILs ranked among the highest five lines for all traits (Table 2).

QTL detection and estimation. QTLs for each trait and their effects were estimated and overlapping QTLs were identified between pairs of traits (Table 5). One region on chromosome 5 (positions 43.3 to $55.3 \mathrm{cM}$ ) and one region on chromosome 8 (positions 95.3 to $103.2 \mathrm{cM}$ ) contained overlapping QTLs affecting the two toxins concentrations. For both toxins, the QTL on chromosome 5 was the largest effect QTL identified. Another region on chromosome 5 (197.1 to $210.1 \mathrm{cM})$ contained overlapping QTLs for the two rots. One very narrow QTL region on chromosome 3 (75.6 to $75.7 \mathrm{cM}$ ) affected three of the four disease traits, Aspergillus rot, fumonisin, and Fusarium rot.

\section{DISCUSSION}

Although both parents of this population exhibited similar levels of rot and mycotoxin contamination resistance, significant

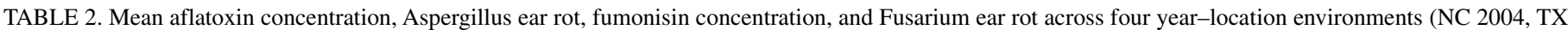
2004, NC 2005, and TX 2005) of recombinant inbred lines (RILs) ranking in the highest (1 to 5) or lowest (44 to 48) five for each trait

\begin{tabular}{|c|c|c|c|c|}
\hline \multirow[b]{2}{*}{ Lines $^{b}$} & \multicolumn{4}{|c|}{ Concentration of mycotoxin and percent rot (rank) ${ }^{\mathrm{a}}$} \\
\hline & Aflatoxin (ng g-1) & Aspergillus ear rot (\%) & Fumonisin $\left(\mu \mathrm{g} \mathrm{g}^{-1}\right)$ & Fusarium ear rot (\%) \\
\hline \multicolumn{5}{|l|}{ In lowest five ${ }^{c}$} \\
\hline RIL-1 (L) & $261(48)$ & $9(46)$ & $5(45)$ & $8(48)$ \\
\hline RIL-82 (L) & $283(47)$ & $12(40)$ & $4(48)$ & $14(40)$ \\
\hline RIL-84 (L) & $295(46)$ & $9(47)$ & $9(36)$ & $9(45)$ \\
\hline RIL-5 (L) & $431(45)$ & $22(27)$ & $11(34)$ & $21(35)$ \\
\hline RIL-68 (L) & $501(44)$ & $9(45)$ & $5(43)$ & $9(47)$ \\
\hline RIL-119 (L) & $1,336(24)$ & $9(48)$ & $21(27)$ & $12(43)$ \\
\hline RIL-67 (L) & $907(32)$ & $10(44)$ & $8(38)$ & $13(41)$ \\
\hline RIL-120 (L) & $556(40)$ & $12(39)$ & $5(47)$ & $12(42)$ \\
\hline RIL-73 (L) & $505(43)$ & $21(33)$ & $5(46)$ & $24(28)$ \\
\hline RIL-135 (L) & $849(33)$ & $11(42)$ & $5(42)$ & $9(46)$ \\
\hline RIL-137 (L) & $939(31)$ & $11(41)$ & $6(39)$ & $11(44)$ \\
\hline RIL-42 (L) & $959(30)$ & $16(36)$ & $5(44)$ & $21(34)$ \\
\hline \multicolumn{5}{|l|}{ In highest five ${ }^{d}$} \\
\hline RIL-48 (L) & $3,285(5)$ & $25(29)$ & $20(28)$ & $28(25)$ \\
\hline RIL-64 (H) & 3,309 (4) & $73(1)$ & $90(5)$ & $81(2)$ \\
\hline RIL-126 (H) & $3,441(3)$ & $41(14)$ & $97(3)$ & $64(6)$ \\
\hline RIL-105 (H) & $3,918(2)$ & $61(3)$ & $111(1)$ & $67(3)$ \\
\hline RIL-113 (H) & $4,876(1)$ & $68(2)$ & $80(6)$ & $85(1)$ \\
\hline RIL-139 (H) & $1,694(18)$ & $57(5)$ & $64(11)$ & $52(12)$ \\
\hline RIL-60 (H) & $2,447(14)$ & $57(4)$ & $80(7)$ & $63(7)$ \\
\hline RIL-80 (H) & $3,225(6)$ & $40(15)$ & $104(2)$ & $32(21)$ \\
\hline RIL-66 (H) & $2,461(13)$ & $45(12)$ & $24(26)$ & $65(5)$ \\
\hline RIL-20 (H) & $1,464(23)$ & $56(6)$ & $90(4)$ & $65(4)$ \\
\hline NC300 (parent) & 122 & 4 & 3 & 7 \\
\hline B104 (parent) & 643 & 25 & 10 & 24 \\
\hline $\operatorname{LSD}(0.05)$ & 1,629 & 21 & 30 & 20 \\
\hline
\end{tabular}

a The rank of the line for each trait is shown within each trait category next to the line name in parentheses.

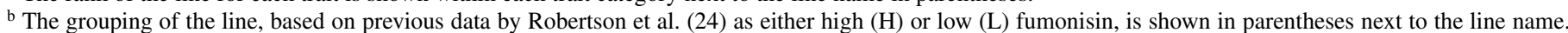
LSD = least significant difference.

${ }^{c}$ Lines ranking in lowest five for at least one trait.

${ }^{\mathrm{d}}$ Lines ranking in highest five for at least one trait.

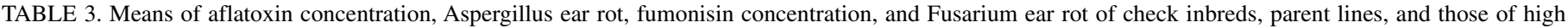
and low selected fumonisin lines within each of the four year-location environments ${ }^{\mathrm{a}}$

\begin{tabular}{|c|c|c|c|c|c|c|c|c|}
\hline \multirow[b]{2}{*}{ Lines $^{d}$} & \multicolumn{4}{|c|}{ Aspergillus flavus $\left(\mathrm{ng} \mathrm{g}^{-1}\right)(\%)^{\mathrm{b}}$} & \multicolumn{4}{|c|}{ Fusarium spp. $\left(\mu \mathrm{g} \mathrm{g}^{-1}\right)(\%)^{\mathrm{c}}$} \\
\hline & NC 2004 & TX 2004 & NC 2005 & TX 2005 & NC 2004 & TX 2004 & NC 2005 & TX 2005 \\
\hline CML176 & $183(2)$ & 313 (14) & $48(0)$ & $53(0)$ & $3(1)$ & $1(19)$ & $0(3)$ & $40(16)$ \\
\hline CML269 & $89(5)$ & $790(10)$ & $98(0)$ & $1,367(20)$ & $12(16)$ & $6(11)$ & $4(3)$ & $11(11)$ \\
\hline CML288 & $61(21)$ & $162(29)$ & $0(\ldots)$ & $3,761(30)$ & $11(9)$ & $4(11)$ & $5(\ldots)$ & $2(39)$ \\
\hline FR2128 & $1,227(15)$ & $211(40)$ & $470(2)$ & $677(25)$ & $36(19)$ & $46(17)$ & $13(5)$ & $29(66)$ \\
\hline TX110 & $244(3)$ & $260(26)$ & $76(2)$ & $493(55)$ & $9(8)$ & $22(26)$ & $1(0)$ & $5(68)$ \\
\hline TX770 & $376(5)$ & $239(20)$ & 778 (2) & $1,582(20)$ & $12(8)$ & $10(35)$ & $1(3)$ & $14(39)$ \\
\hline NC300 & $214(2)$ & $165(10)$ & $36(0)$ & $0(6)$ & $4(2)$ & $2(13)$ & $1(1)$ & $4(10)$ \\
\hline B104 & $285(3)$ & $710(18)$ & $408(2)$ & $1,076(73)$ & $10(7)$ & $10(23)$ & $3(3)$ & $16(61)$ \\
\hline High mean & $1,036(23)$ & $3,440(64)$ & $1,248(11)$ & $3,629(80)$ & $65(41)$ & $70(64)$ & $41(16)$ & $68(75)$ \\
\hline Low mean & $302(7)$ & $743(22)$ & $440(2)$ & $2,031(38)$ & $12(11)$ & $11(22)$ & $5(4)$ & $18(34)$ \\
\hline High-low mean & $734(16)$ & $2,697(42)$ & $808(9)$ & $1,598(42)$ & $53(30)$ & $59(42)$ & $36(12)$ & $50(41)$ \\
\hline Contrasts & $* * * *(* * * *)$ & $* * * *(* * * *)$ & $* * * *(* * * *)$ & $*(* * * *)$ & $* * * *(* * * *)$ & $* * * *(* * * *)$ & $* * * *(* * * *)$ & $* * * *(* * * *)$ \\
\hline $\operatorname{LSD}(0.05)^{\mathrm{e}}$ & $746(15)$ & $1,305(20)$ & $853(8)$ & $3,743(34)$ & $40(17)$ & $44(17)$ & $27(7)$ & $50(32)$ \\
\hline
\end{tabular}

${ }^{a}$ Results of comparing selected high- and low-fumonisin groups for each trait using contrasts also are shown.

${ }^{b}$ Means are aflatoxin concentration $\left(\mathrm{ng} \mathrm{g}^{-1}\right)$, followed by percent Aspergillus ear rot.

${ }^{c}$ Means are fumonisin concentration $\left(\mu \mathrm{g} \mathrm{g}^{-1}\right)$, followed by percent Fusarium ear rot.

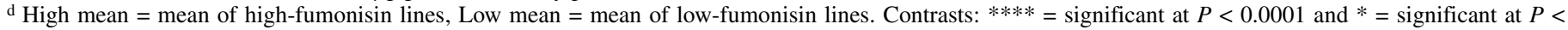
0.05 .

e Least significant differences (LSDs) are appropriate for comparing any two line means. 
segregation was observed in the population, illustrated by the range of line means (Tables 1 and 2).

The two groups of RILs, selected based on high and low fumonisin levels from a previous experiment, were significantly different for all four traits in the four environments (Table 3), suggesting that selection in the RILs for reduced fumonisin concentration based on three North Carolina environments was significant in independent Texas and North Carolina environments. This also suggests that genotype-environment interaction for fumonisin contamination is due mainly to differences in genetic variance for fumonisin within environments, rather than to significant changes in genotype ranks (23). Finally, the correlated response of Fusarium and Aspergillus ear rots and aflatoxin concentration to divergent selection on fumonisin concentration strongly implicates a genetic correlation between fumonisin concentration and the other disease traits.

The relationship of resistance to both mycotoxin contaminations and ear rots is also supported directly by the correlation estimates. Both genotypic and phenotypic correlations between all pairs of traits were high (Table 4). However, the genotypic correlations were always greater than the phenotypic correlations, suggesting that environmental factors reduce the extent to which these traits are phenotypically correlated. A caveat to this conclusion is that the genotypic correlation estimates may be biased by the selection for extreme mean fumonisin concentrations based on a previous study.

Because of the high correlations between all traits across environments, the significant differences of the means of the selected high- and low-fumonisin groups for all traits, and the high genotypic correlation between fumonisin contamination and Fusarium ear rot (23), we predict that making selections against Fusarium ear rot also would select lines with lower fumonisin contamination. Similarly, the strong correlations between Fusarium and Aspergillus ear rots and between Aspergillus ear rot and aflatoxin contamination observed in this study suggest that selection against Fusarium ear rot also should result in reduced susceptibility to Aspergillus ear rot and aflatoxin contamination. This is a favorable situation because Fusarium ear rot is easy to score in the field, relatively stable across environments, and much less expensive to phenotype than mycotoxin contamination.

Although the estimated effects of the QTLs identified are biased upward due to their identification in a small and nonrandom set of lines, the consistency of QTLs identified between the traits supports the correlations estimated between all four of the traits in the population. None of the QTLs, however, is large enough to explain the very high correlations calculated. This may be due to the small sample size used, which limits the power to detect QTLs (24). The limited similarity between QTLs identified for Fusarium ear rot and fumonisin in this subsample of lines and in the overall population in a previous study (25) is evidence that the small sample of RILs in this study was not sufficient for whole-genome QTL scanning. Only three fumonisin QTLs (located on chromosomes 3, 6, and 8) and one Fusarium ear rot QTL (chromosome 3) detected in this study (Table 5) were consistent with QTLs identified using the entire population (25). However, of the three fumonisin QTLs identified in this study consistent with the QTLs in the overall study, the QTL on chromosome 6 was the second largest effect QTL identified in the overall study, and one Fusarium ear rot QTL identified in this study was consistent with the second largest effect QTL in the overall study (Table 5) (25). These consistent QTLs, identified in two separate studies of the same population, provide additional evidence that these chromosomal regions contain genes associated with resistance. Fusarium ear rot and fumonisin resistance QTLs were consistent with our past findings; therefore, the locations of QTLs for Aspergillus ear rot and aflatoxin identified in this study are supported (25).

The lack of overlap between the fumonisin QTLs identified in the original full population study and the aflatoxin QTL study performed by Paul et al. (19) could be interpreted as evidence against common QTL regions for the two mycotoxin phenotypes. However, even within the Paul et al. (19) study, very few QTLs overlapped in the two populations in the two environments studied (1996 and 1997 in Urbana, IL), suggesting that environment plays a large role in determining aflatoxin contamination phenotype and that different populations contain different resistance QTLs.

TABLE 5. Quantitative trait loci (QTLs) identified through multiple interval mapping for the four traits (aflatoxin, Aspergillus ear rot, fumonisin, and Fusarium ear rot) in the selected recombinant inbred lines

\begin{tabular}{|c|c|c|c|}
\hline Chromosome & $\begin{array}{l}\text { QTL position } \\
(\mathrm{cM})^{\mathrm{a}}\end{array}$ & $\begin{array}{c}\text { Effect } \\
\text { (concentration) }^{\mathrm{b}}\end{array}$ & $\begin{array}{c}\text { Phenotypic } \\
\text { variation } \\
\text { explained (\%) }\end{array}$ \\
\hline \multicolumn{4}{|l|}{ Aflatoxin } \\
\hline 1 & 148.6 & 528 & 21.6 \\
\hline 5 & 43.3 & -729 & 45.4 \\
\hline 8 & 103.2 & 400 & 6.6 \\
\hline 9 & 31.3 & -235 & 5.6 \\
\hline 10 & 51.9 & 310 & 12.5 \\
\hline Total variation $^{\mathrm{c}}$ & $\ldots$ & $\ldots$ & 91.7 \\
\hline \multicolumn{4}{|l|}{ Aspergillus rot } \\
\hline 3 & 7.5 & 4 & 3.6 \\
\hline 3 & 75.7 & 9 & 20.9 \\
\hline 4 & 21.0 & 4 & 7.8 \\
\hline 5 & 197.1 & 6 & 16.0 \\
\hline 6 & 140.0 & -8 & 20.8 \\
\hline Total variation $^{\mathrm{c}}$ & $\ldots$ & $\ldots$ & 69.1 \\
\hline \multicolumn{4}{|l|}{ Fumonisin } \\
\hline 3 & 75.6 & 10 & 11.6 \\
\hline 5 & 55.3 & -21 & 46.1 \\
\hline 6 & 140.0 & -13 & 18.7 \\
\hline 8 & 95.3 & 10 & 8.2 \\
\hline Total variation $^{\mathrm{c}}$ & $\ldots$ & $\ldots$ & 84.6 \\
\hline \multicolumn{4}{|l|}{ Fusarium rot } \\
\hline 3 & 75.6 & 8 & 15.3 \\
\hline 5 & 210.1 & 7 & 18.2 \\
\hline 8 & 197.9 & -7 & 3.1 \\
\hline 10 & 41.3 & 12 & 40.8 \\
\hline $3 \times 5^{\mathrm{d}}$ & $\ldots$ & -2 & 3.2 \\
\hline $3 \times 8^{d}$ & $\ldots$ & -5 & 5.6 \\
\hline Total variation $^{\mathrm{c}}$ & $\ldots$ & $\ldots$ & 86.2 \\
\hline
\end{tabular}

${ }^{a}$ Position of each QTL is in centimorgans (cM).

${ }^{\mathrm{b}}$ QTL effect estimated as half the difference between homozygous NC300 and homozygous B104 genotypes. Effect is in $\mathrm{ng} \mathrm{g}^{-1}$ for aflatoxin, $\mu \mathrm{g} \mathrm{g}^{-1}$ for fumonisin, and percentage for Aspergillus and Fusarium ear rot. Positive effects refer to B104 as the origin of the beneficial allele, and negative effects refer to $\mathrm{NC} 300$ as the beneficial allele.

${ }^{c}$ Total variation associated with all of the QTLs in the model fitted simultaneously.

${ }^{\mathrm{d}}$ Epistatic interactions are presented using the chromosome to designate which QTL is involved in the interaction.

TABLE 4. Genotypic and phenotypic correlation estimates (and their standard errors) between the four disease traits ${ }^{\mathrm{a}}$

\begin{tabular}{lcccc}
\hline & Fumonisin & Aflatoxin & Fusarium ear rot & Aspergillus ear rot \\
\hline Fumonisin & $\ldots$ & $0.95(0.06)$ & $0.92(0.04)$ & $0.91(0.05)$ \\
Aflatoxin & $0.53(0.06)$ & $\ldots$ & $0.94(0.05)$ & $0.88(0.06)$ \\
Fusarium ear rot & $0.67(0.05)$ & $0.63(0.05)$ & $\ldots$ & $0.99(0.01)$ \\
Aspergillus ear rot & $0.60(0.05)$ & $0.63(0.05)$ & $0.81(0.03)$ & $\ldots$ \\
\hline
\end{tabular}

${ }^{\text {a }}$ Genotypic correlation estimates are presented above the diagonal and the phenotypic correlation estimates are shown below the diagonal. 
Although we did not identify individual RILs with significantly lower toxin or rot levels than the parent lines, we did identify RILs that had significantly higher toxin and rot levels than either parent (Table 2), indicating that the two parents had complementary sets of resistance alleles. The fact that we did not see RILs with better resistance than either parent suggests epistasis in the genetic control of resistance, whereby resistance alleles act in a less than additive fashion. Thus, individual resistance genes have greater effects individually than in multiple-locus combinations. However, in the QTL analysis, epistasis was identified only for Fusarium ear rot, indicating that the sample size was far too small to estimate epistatic effects reliably.

Our results suggest the existence of some common resistance mechanisms to these two fungi, but many questions remain to be addressed to further elucidate these relationships. The possibility of common host resistance mechanisms of maize, as well as common infection and virulence mechanisms of the fungi, should be investigated. Other factors that impact that fungus-plant interaction, such as insect resistance or damage, also should be considered. Abbas et al. (1) found that both Aspergillus and Fusarium ear rot were significantly correlated with insect damage. Also, genes that control plant stress reactions also may contribute to the correlation between resistances to these two fungi. For example, stress due to weather conditions influences both fumonisin and aflatoxin contamination $(2,20)$.

One approach to investigating the relationship between resistances to the ear rot pathogens is QTL mapping. QTLs for different disease resistances have some tendency to map to similar genome locations (34). Localizing QTLs for resistance to different diseases on a common genetic map provides circumstantial evidence for common genetic mechanisms. Fine-scale genetic mapping will be necessary to distinguish linked QTLs, such as those in a resistance "cluster," from pleiotropic QTLs that influence resistance to both fungi. When comparing the RobertsonHoyt et al. QTL data (25) to the data synthesized by Wisser et al. (34), for example, several fumonisin contamination QTLs were consistent with QTLs for aflatoxin contamination on chromosomes $1,2,3,4,5$, and 9. These results, along with the high positive correlations between the four traits reported in this study, suggest that at least some of the genetic factors are linked or have identical pleiotropic QTLs. In either case, these QTL clusters can be exploited by plant scientists looking for resistance to multiple fungal species. Although host resistance to these traits is complex, with different sets of resistance factors in different lines, it appears that some resistance QTL regions may have pleiotropic effects on Fusarium ear rot, Aspergillus ear rot, fumonisin, and aflatoxin.

\section{ACKNOWLEDGMENTS}

This research was supported by a U.S. Department of Agriculture IFAFS multidisciplinary training grant to North Carolina State University (award 2001-52101-11507) and by a grant from the Texas Corn Producers Board. We thank M. Goodman for the RIL population; B. Peterson and S. Salvo for technical assistance; and M. Starr, T. Flint, A. Hunt, S. Reed, and N. Coles for field inoculation assistance.

\section{LITERATURE CITED}

1. Abbas, H. K., Cartwright, R. D., Xie, W., and Shier, W. T. 2006. Aflatoxin and fumonisin contamination of corn (maize, Zea mays) hybrids in Arkansas. Crop Prot. 25:1-9.

2. Abbas, H. K., Williams, W. P., Windham, G. L., Pringle, H. C., Xie, W., and Shier, W. T. 2002. Aflatoxin and fumonisin contamination of commercial corn (Zea mays) hybrids in Mississippi. J. Agric. Food Chem. 50:5246-5254.

3. Cardinal, A. J., and Lee, M. 2005. Genetic relationships between resistance to stalk-tunneling by the European corn borer and cell-wall components in maize population B73 $\times$ B52. Theor. Appl. Genet. 111:1-7.

4. Castegnaro, M., and McGregor, D. 1998. Carcinogenic risk assessment of mycotoxins. Rev. Med. Vet. 149:671-678.

5. CFSAN. 2000. Action levels for poisonous or deleterious substances in human food and animal feed. Published online by the U.S. Food and Drug Administration Center for Food Safety and Applied Nutrition.

6. CFSAN. 2001. Background paper in support of fumonisin levels in corn and corn products intended for human consumption. Published online by the U.S. Food and Drug Administration Center for Food Safety and Applied Nutrition and the Center for Veterinary Medicine.

7. CFSAN. 2001. Guidance for industry: Fumonisin levels in human foods and animal feeds. Published online by the U.S. Food and Drug Administration Center for Food Safety and Applied Nutrition and the Center for Veterinary Medicine.

8. Churchill, G. A., and Doerge, R. W. 1994. Empirical threshold values for quantitative trait mapping. Genetics 138:963-971.

9. Clements, M. J., Kleinschmidt, C. E., Maragos, C. M., Pataky, J. K., and White, D. G. 2003. Evaluation of inoculation techniques for Fusarium ear rot and fumonisin contamination of corn. Plant Dis. 87:147-153.

10. Colvin, B. M., and Harrison, L. R. 1992. Fumonisin-induced pulmonary edema and hydrothorax in swine. Mycopathologia 117:79-82.

11. Gelderblom, W. C. A., Jaskiewicz, K. Marasas, W. F. O. Thiel, P. G. Horak, R. M., Vleggaar, R., and Kriek, N. P. J. 1988. Fumonisins-novel mycotoxins with cancer-promoting activity produced by Fusarium moniliforme. Appl. Environ. Microbiol. 54:1806-1811.

12. Hendricks, K. 1999. Fumonisins and neural tube defects in south Texas. Epidemiology 10:198-200.

13. Holland, J. B. 2006. Estimating genotypic correlations and their standard errors using multivariate restricted maximum likelihood estimation in SAS Proc MIXED. Crop Sci. 46:642-654.

14. Holland, J. B., Nyquist, W. E., and Cervantes-Martinez, C. T. 2003. Estimating and interpreting heritability for plant breeding: An update. Plant Breed. Rev. 22:9-112.

15. Littell, R. C., Milliken, G. A., Stroup, W. W., and Wolfinger, R. D. 1996. SAS System for Mixed Models. SAS Institute Inc., Cary, NC.

16. Marín, S., Sanchis, V., Ramos, A. J., Vinas, I., and Magan, N. 1998. Environmental factors, in vitro interactions, and niche overlap between Fusarium moniliforme, F. proliferatum, and F. graminearum, Aspergillus and Penicillium species from maize grain. Mycol. Res. 102:831-837.

17. Melchinger, A. E., Utz, F. H., and Schön, C. C. 1998. Quantitative trait locus (QTL) mapping using different testers and independent population samples in maize reveals low power of QTL detection and large bias in estimates of QTL effects. Genetics 149:383-403.

18. Ono, E. Y. S., Ono, M. A., Funo, F. Y., Medina, A. E., Oliveira, T. C. R. M., Kawamura, O., Ueno, Y., and Hirooka, E. Y. 2001. Evaluation of fumonisin-aflatoxin co-occurrence in Brazilian corn hybrids by ELISA. Food Addit. Contam. 18:719-729.

19. Paul, C., Niadoo, G., Forbes, A., Mikkilineni, V., White, D., and Rocheford, T. 2003. Quantitative trait loci for low aflatoxin production in two related maize populations. Theor. Appl. Genet. 107:263-270.

20. Payne, G. A. 1992. Aflatoxin in maize. Crit. Rev. Plant Sci. 10:423-440.

21. Piepho, H. P., and Gauch, H. G. 2001. Marker pair selection for mapping quantitative trait loci. Genetics 157:433-444.

22. Rheeder, J. P., Marasas, W. F. O., Thiel, P. G., Sydenham, E. W., Shephard, G. S., and van Schalkwyk, D. J. 1992. Fusarium moniliforme and fumonisins in corn in relation to human esophageal cancer in Transkei. Phytopathology 82:353-357.

23. Robertson, L. A., Kleinschmidt, C. E., White, D. G., Payne, G. A., Maragos, C. M., and Holland, J. B. 2006. Heritabilities and correlations of Fusarium ear rot resistance and fumonisin contamination resistance in two maize populations. Crop. Sci. 46:353-361.

24. Robertson, L. A., Payne, G. A., and Holland, J. B. 2005. Marker assisted breeding for host resistance to mycotoxin contamination. Pages 423-435 in: Aflatoxin and Food Safety. H. K Abbas, ed. Marcel Dekker, New York.

25. Robertson-Hoyt, L. A., Jines, M. P., Balint-Kurti, P. J., Kleinschmidt, C. E., White, D. G., Payne, G. A., Maragos, C. M., Molnár, T. L., and Holland, J. B. 2006. QTL mapping for Fusarium ear rot and fumonisin contamination resistance in two maize populations. Crop Sci. 46:17341743.

26. Ross, P. F., Rice, L. G., Osweiler, G. D., Nelson, P. E., Richard, J. L., and Wilson, T. M. 1992. A review and update of animal toxicoses associated with fumonisin-contaminated feeds and production of fumonisins by Fusarium isolates. Mycopathologia 117:109-114.

27. SAS Institute. 1999. SAS online doc version eight. SAS Institute, Cary, NC.

28. Self, S. G., and Liang, K. 1987. Asymptotic properties of maximum likelihood estimators and likelihood ratio tests under nonstandard conditions. J. Am. Stat. Assoc. 82:605-610.

29. Senior, M. L., Murphy, J. P., Goodman, M. M., and Stuber, C. W. 1998. Utility of SSRs for determining similarities and relationships in maize using an agarose gel system. Crop Sci. 38:1088-1098.

30. Visscher, P. M., Thompson, R., and Haley, C. S. 1996. Confidence 
intervals in QTL mapping by bootstrapping. Genetics 143:1013-1020.

31. Wang, S., Basten, C. J., and Zeng, Z.-B. 2006. Windows QTL Cartographer 2.5. Available online. Department of Statistics, North Carolina State University, Raleigh, NC.

32. Wicklow, D. T., Norton, R. A., and McAlpin, C. E. 1998. Beta-carotene inhibition of aflatoxin biosynthesis among Aspergillus flavus genotypes from Illinois corn. Mycoscience 39:167-172.

33. Windham, G. L., Williams, W. P., Buckley, P. M., and Abbas, H. K. 2003.
Inoculation techniques used to quantify alflatoxin resistance in corn. J. Toxicol. Toxin Rev. 22(2\&3):313-325.

34. Wisser, R. J., Balint-Kurti, P. J., and Nelson, R. J. 2006. The genetic architecture of disease resistance in maize: A synthesis of published studies. Phytopathology 96:120-129.

35. Zummo, N., and Scott, G. E. 1992. Interaction of Fusarium moniliforme and Aspergillus flavus on kernel infection and aflatoxin contamination in maize ears. Plant Dis. 76:771-773. 